\title{
The role of temporal context in norm-based encoding of faces
}

\author{
Bram Van Rensbergen • Hans P. Op de Beeck
}

Published online: 26 July 2013

(C) Psychonomic Society, Inc. 2013

\begin{abstract}
Research shows that the human brain encodes faces in terms of how they relate to a prototypical face, a phenomenon referred to as norm-based encoding. The goal of this study was to examine the effect of short-term exposure on the development of the norm, independently of global, long-term exposure. We achieved this by varying the sequence of presentation of the stimuli while keeping global exposure constant. We found that a systematic manipulation of the average face in a set of 10 preceding trials can shift this norm toward that average. However, there was no effect of order or recency among these trials; thus, there was no evidence that the last faces mattered more than the first. This suggests that the position of the face norm is modified by information that is integrated across multiple recent faces.
\end{abstract}

\section{Keywords Face perception $\cdot$ Categorization $\cdot$ Similarity}

How do people distinguish one face from another? How do we store a face in our brain? Even though there has been extensive research on these topics allowing a partial answer to these questions, many details remain unknown. Recent research in a wide variety of fields shows that the human brain represents objects and faces using a norm-based encoding system (e.g., Lee, Byatt, \& Rhodes, 2000; Leopold, Bondar, \& Giese, 2006; Leopold, O'Toole, Vetter, \& Blanz, 2001; Leopold, Rhodes, Müller, \& Jeffery, 2005; Loffler, Yourganov, Wilkinson, \& Wilson, 2005; Nishimura, Maurer, Jeffery, Pellicano, \& Rhodes, 2008; Op de Beeck, Wagemans, \& Vogels, 2003; Panis, Wagemans, \& Op de Beeck, 2011; Rhodes \& Jeffery, 2006; Suzuki \& Cavanagh,

Electronic supplementary material The online version of this article (doi:10.3758/s13423-013-0478-0) contains supplementary material, which is available to authorized users.

B. Van Rensbergen · H. P. Op de Beeck $(\bowtie)$

Department of Psychology, University of Leuven, Tiensestraat 102

B3714, 3000 Leuven, Belgium

e-mail: hans.opdebeeck@ppw.kuleuven.be
1998). Here, we extend these findings and investigate how the position of the underlying norm is influenced by the history of presented faces.

In a norm-based encoding system, faces are stored as their deviation from a prototypical face or norm. Yet research indicates that the norm itself is not static, but changes as we are presented with new information; more specifically, the norm appears to move toward recently presented stimuli (e.g., Carbon et al., 2007; Leopold et al., 2005; Nishimura et al., 2008; Panis et al., 2011).

If faces are stored as deviations from a norm, changes to that norm would alter the appearance of subsequently viewed faces, because their deviation from the norm would change. Research shows that this is indeed true, since changes to the norm are reported to alter perceived normality (Rhodes, Jeffery, Watson, Clifford, \& Nakayama, 2003), identification (Leopold et al., 2001), and perceived attractiveness (Rhodes et al., 2003).

Such effects of experience on the norm can work on different time scales. In this study, we investigated the effect of short-term exposure on the development of the norm, independently of global, long-term exposure. We did this by varying the sequence of presentation of the stimuli while keeping global exposure constant. We investigated three different time scales by looking at the effect of (1) the global average of all faces in experimental blocks, (2) the average of the faces on the last 10 trials, and (3) the recency of faces on these last 10 trials.

In our investigation, we deduced the position of the current face norm from the asymmetry of similarity judgments. Indeed, when two stimuli are compared, the order of presentation matters. For example, most people agree that 99 resembles 100 more than 100 resembles 99. In general, the similarity between a special stimulus (e.g., the norm) and a "regular" stimulus is considered larger when the special stimulus is presented second than when the special stimulus is presented first (Nosofsky, 1991; Tversky, 1977). In our study, we used this asymmetry effect as a measure of how 
close a stimulus was to the norm. We could then use this measurement to investigate the influence of different time scales on the development of the norm.

\section{Method \\ Participants}

A total of 35 first-year psychology students of the University of Leuven participated in return for course credit. Data of 2 participants were not analyzed due to a technical issue during data acquisition. All participants signed a written informed consent form.

\section{Stimuli}

An essential aspect of our study was being able to attribute any effects of the norm to its special status and to dissociate these effects from the norm's physical characteristics. To that end, we created four sets of stimuli.

We started from four photographs of faces retrieved from online photo databases. Adobe Photoshop CS 4 was used to create alternative versions of the photos by making some of its features more extreme (see Fig. 1 for an example). Changes were made to the location and size of the mouth, eyes, eyebrows, and nose. The extent and direction of these changes was not related to the original features of the face; that is, we were not caricaturizing the face.

We then used morphing software WinMorph to create four morph lines. For two morph lines (A and B in Fig. 2), we morphed between the symmetrical original face and an extreme version of that same face, resulting in a total of seven versions of the same image that gradually changed from normal to extreme.

For the two other morph lines, we created two different extreme versions of the original face, one extreme being the opposite of the other extreme on the manipulated feature
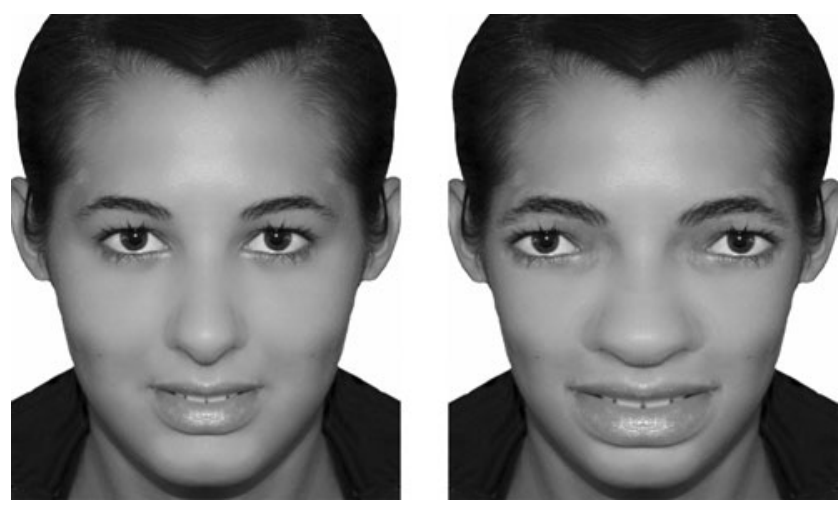

Fig. 1 An example of an original and an extreme face. A symmetrical but otherwise unaltered face (left), and its 'extreme' version (right) dimensions (sets $\mathrm{C}$ and $\mathrm{D}$ in Fig. 2). For these morph lines, we morphed between the two different extreme versions, resulting in seven versions of the same image that gradually changed from one extreme to the other.

We included these two types of morph lines, normalextreme and extreme-extreme, to deconfound the norm from the original photo. For the normal-extreme morph lines, the original photo was a distant face, far from the average of the other faces. In contrast, for the extreme-extreme morph lines, we morphed between two opposing extremes of the original face, and consequently, the original photo was closest to the middle of all seven faces.

\section{Procedure}

A trial always consisted of two photos of the same morph line. First, a fixation cross $(306 \mathrm{~ms})$ and the first photo (again, $306 \mathrm{~ms}$ ) were shown at the exact center of the screen. After this, a second fixation cross (306 ms) and the second photo (306 ms) were shown, again at the center of the screen. Participants were then asked to rate the difference between the two images on a scale of 1 to 7 , where 1 corresponded to no perceived difference and 7 to a large perceived difference (responses were given through a keyboard). Stimuli were displayed on an $85-\mathrm{Hz} 17$-in. gamma-corrected screen in a darkened room, using a Toshiba laptop running PsychToolbox (Brainard, 1997). Stimulus size was approximately $8^{\circ}$ (horizontal) $\times 10^{\circ}$. Participants were seated at approximately $70 \mathrm{~cm}$; no chinrest was used.

The experiment consisted of eight blocks, two for each morph line (two blocks of one particular morph line were always presented successively). The order of these four groups of two blocks was counterbalanced between participants. Each block contained 60 trials. Each participant saw only five of the seven stimuli of any particular morph line; each participant was presented with two morph lines that used the first five photos and two morph lines that contained the last five photos. Which of the morph lines used the first five faces and which of the blocks used the last five faces was counterbalanced between participants.

Of the five stimuli of a morph line that a participant saw in one block, the middle image (face 3 in Fig. 3) always lay halfway between the other four faces on the morphing continuum and, thus, possessed intermediate facial features. However, recall that some participants saw the first five of the seven stimuli of a morph line, while others saw the last five. This means that which faces possessed intermediate features and which faces possessed "extreme" features differed between participants; only their relative positions remained constant. When we use the term mid-face throughout this text, we will be referring to the middle image for that participant and that morph line (e.g., F3 in Fig. 3). When we 
Fig. 2 An overview of all the used stimuli. Two of the sets were morphed from normal to extreme (a and $\mathbf{b}$ ), and two from extreme to extreme (c and $\mathbf{d}$ ). Any given participant saw only five out of seven images of each morph line, either the first five, or the last five

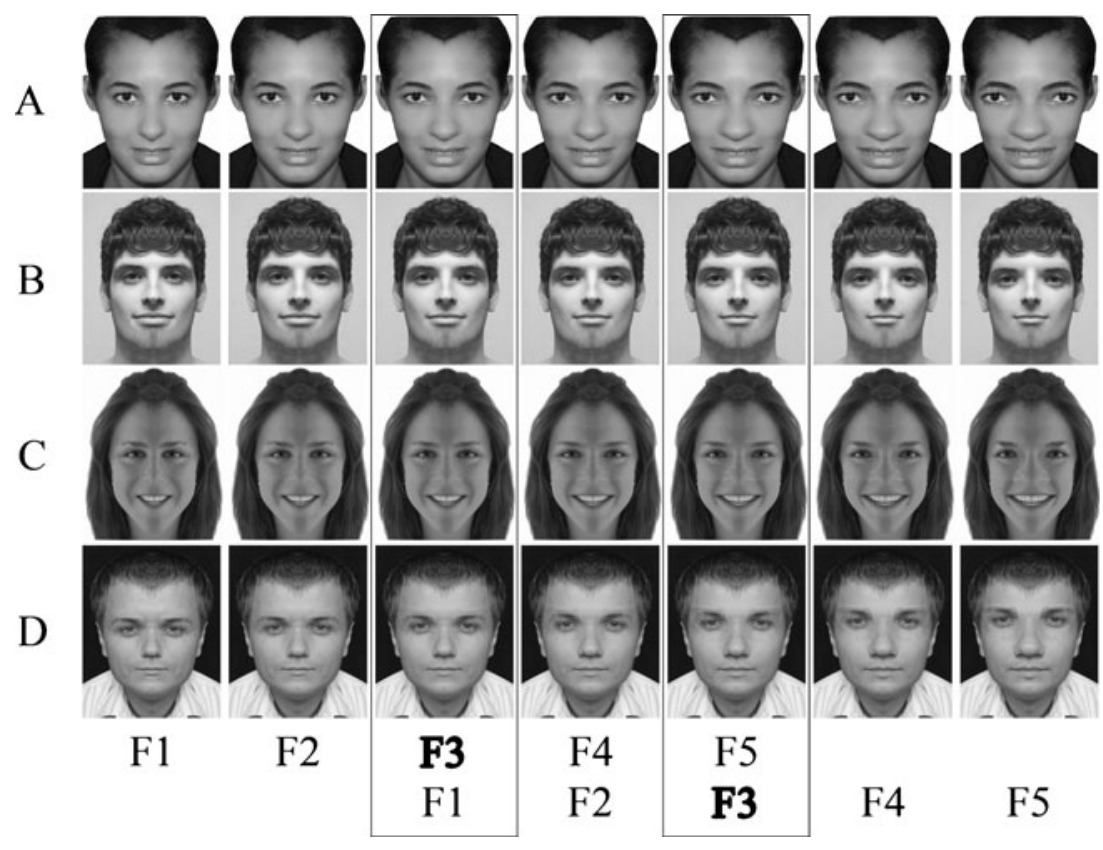

use the term distant face, we refer to the outer faces for that participant and that morph line (e.g., F1 and F5 in Fig. 3).

Each of the 15 possible combinations of items was presented 4 times in one block. The three combinations with features that averaged to the mid-face (i.e., F1-F5, F2-F4, and F3-F3 trials, using the numerical index from Figs. 2 and 3 ) were not useful for our manipulations, and as such, those $12(3 \times 4)$ trials were presented at the start of the block, to induce a global context.

For each particular participant, global exposure to the stimuli was kept constant throughout the entire experiment. This was done by making sure that every photo used in the experiment was seen equally often. In practice, this meant that each of the 20 photos that were used ( 5 for each of the four morph lines) was presented 48 times.

Presentation order of the stimuli was manipulated in several ways. The basic concept was that each block was divided into sets of six trials. Each set consisted of five contextsetting trials and one trial of interest, the test trial.

Three experimental manipulations were made: the order of presentation on the test trial (to measure asymmetry), the distance of the total average across all 10 stimuli on the five context-setting trials to the average position of the two stimuli on the test trial, and whether the averages of the two stimuli from each of the five context-setting trials moved toward the mid-face or away from it. We will discuss all three manipulations in detail.

The first manipulation, presentation order, was introduced to measure the asymmetry effect. The test trial always consisted of the mid-face (F3) and a distant face (F1 or F5). On the basis of previous research (Op de Beeck et al., 2003; Panis et al., 2011), we expected to find a higher perceived similarity when the mid-face was presented after the distant face than when it was presented before the distant face.

The second manipulation, contextual average, was introduced to measure the effect of the content of the contextsetting trials on the measured asymmetry on the following test trials. The stimuli used on the five context-setting trials were chosen in such a way that the averages of each trial were either all below or all above the mid-face for a particular participant. After the context-setting trials, a test trial was presented. Recall that the test trial always consisted of the mid-face and a distant face. This setup yields two possibilities:
Fig. 3 An example of stimuli used in a particular block. The stimuli range from distant (F1) over midface (F3) to distant (F5) again

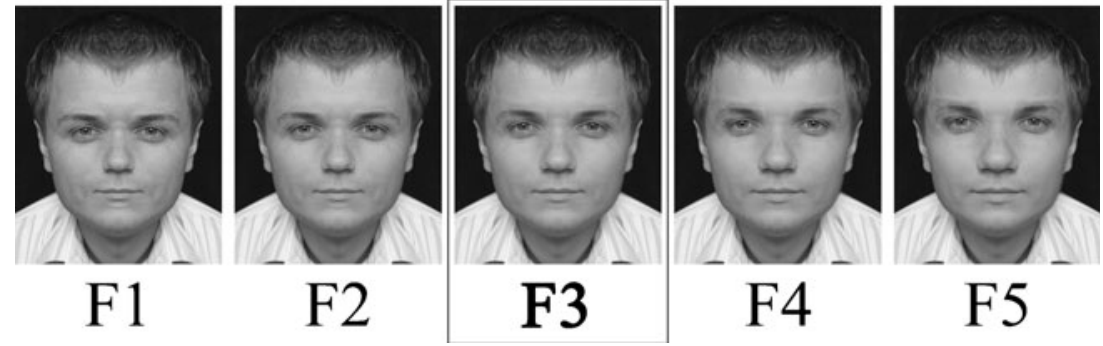


1. Close history: The stimuli within the context-setting trials are, on average, relatively close to the distant face on the test trial. For example, context-setting trials include stimuli with low numbers on a morph line (e.g., F1 and F2), and face F1 is used as the distant face on the test trial, or, alternatively, context-setting trials include stimuli with high numbers on a morph line (e.g., F4 and F5), and face F5 is used as the distant face on the test trial.

2. Far history: The stimuli within the context-setting trials are, on average, relatively far from the distant face on the test trial. For example, context-setting trials include stimuli with low numbers on a morph line and face F5 is used as the distant face on the test trial, or contextsetting trials include stimuli with high numbers on a morph line and face F1 is used as the distant face on the test trial.

In a far history context, the context-setting trials shift the norm away from the distant face used on the consequent test trial, making that distant face look even more distant. This means that, in a far history context, the difference in prototypicality between the mid-face and the distant face is expected to remain very large. This is in contrast to close history context, where the averages of the context-setting trials lie between the mid-face and the distant face used on the test trial. This makes those two more similar in prototypicality. Consequently, we predict a smaller asymmetry effect for close history contexts than for far history contexts.

The third manipulation, contextual trend, was introduced to examine the effect of recency on the development of the norm. For each set of five context-setting trials, the averages moved either toward or away from the mid-face. We predicted that this manipulation would affect the norm and interact with the manipulation of close history versus far history contexts, but only if recency matters and more recent stimuli have more impact on the norm on the test trial. In that case, a close history context moving toward the mid-face should emphasize the norm-like status of this mid-face, since the most recent stimuli are more similar to this mid-face than to the distant face on the test trial. In contrast, a close history context moving away from the mid-face and, thus, toward the distant face would decrease the norm-like status of the mid-face, since the most recent stimuli are more similar to the distant face than to the mid-face. Thus, we predicted a strong effect of recency in the close history contexts. In far history contexts, the prediction is less clear, since both the mid-face and the distant face on the test trial fall at the same side of the morph line, as compared with the stimuli on the context-setting trials. In that case, any order that would move away from the mid-face would also move away from the distant face on the test trial. In sum, if we hypothesized that more recent stimuli would have a stronger impact on the position of the norm, we would predict a larger asymmetry effect when the preceding trials moved toward the mid-face than when the preceding trials moved away from the midface, at least for the close history contexts.

In conclusion, three manipulations of two possibilities each were made, resulting in a $2 \times 2 \times 2$ design (see Table 1 for an overview of all manipulations; see Table 2 for an example of sets of stimuli varying in contextual average and contextual trend; and refer to the Supplemental Materials for an example of a complete block of trials including all $2 \times 2 \times 2$ conditions).

\section{Results}

Data for the dissimilarity judgments were collected and analyzed using a repeated measures general linear model. Three within-subjects variables were defined, each with two values: presentation order of items on the test trial (global average first or global average second), contextual average (close history or far history), and contextual trend (moving toward the global average or moving away from it). We tested for the three main effects and all possible interactions (refer to Table 3 for an overview of the mean difference ratings in all eight $(2 \times 2 \times 2)$ possible conditions and to Table 4 for an overview of the statistical tests).

In line with our predictions, we found a significant main effect of test trial order, $F(1,32)=10.623, p=.003, \eta^{2}=.249$, showing that whether the global average (the mid-face) was presented first or second on the test trial influenced difference ratings. As was expected, the difference between the global average and the other stimulus was judged as larger when the global average was presented first than when it was presented second (see Fig. 4 for an illustration of this effect).

Table 1 A summary of the manipulations

\begin{tabular}{lll}
\hline Manipulation & First Possibility & Second Possibility \\
\hline $\begin{array}{l}\text { Presentation order } \\
\text { Contextual average }\end{array}$ & $\begin{array}{l}\text { Mid-face is presented first in the trial of interest } \\
\text { Close history: Contextual average is close to the } \\
\text { distant face of the trial of interest } \\
\text { Recent trials move toward the mid-face }\end{array}$ & $\begin{array}{l}\text { Mid-face is presented second in the trial of interest } \\
\text { Far history: Contextual average is far from the distant } \\
\text { face of the trial of interest }\end{array}$ \\
Contextual trend & Recent trials move away from the mid-face
\end{tabular}


Table 2 An illustration of possible sets of stimuli varying in contextual average and contextual trend

\begin{tabular}{|c|c|c|c|c|c|c|c|}
\hline \multirow{2}{*}{$\begin{array}{l}\text { Contextual Average } \\
\text { Close history }\end{array}$} & \multirow{2}{*}{$\begin{array}{l}\text { Contextual Trend } \\
\text { Trials move toward the mid-face }\end{array}$} & \multicolumn{5}{|c|}{ Context-Setting Trials } & \multirow{2}{*}{$\begin{array}{l}\text { Test Trial } \\
\text { F5-F3 }\end{array}$} \\
\hline & & F5-F5 & F4-F5 & F4-F4 & F2-F5 & F3-F4 & \\
\hline & Trials move away from the mid-face & F2-F3 & F4-F1 & $\mathrm{F} 2-\mathrm{F} 2$ & $\mathrm{~F} 1-\mathrm{F} 2$ & F1-F1 & F1-F3 \\
\hline \multirow[t]{2}{*}{ Far history } & Trials move toward the mid-face & F1-F1 & F1-F2 & F2-F2 & F4-F1 & F3-F2 & F3-F5 \\
\hline & Trials move away from the mid-face & F4-F3 & F5-F2 & F4-F4 & F5-F4 & F5-F5 & F1-F3 \\
\hline
\end{tabular}

Again, as was predicted, we found a significant interaction between the average of the context-setting trials and the test trial order, $F(1,32)=11.633, p=.002, \eta^{2}=.267$ (see Fig. 4). For close history contexts, presentation order seems to have no influence on dissimilarity ratings, whereas for far history contexts, trials where the prototype is presented first yield a much higher dissimilarity rating than do trials where the prototype is presented last. This finding indicates that the norm moves toward the average of the stimuli presented on the context-setting trials. Consequently, no asymmetry was present when the contextual average was in the middle of the two stimuli on the test trial (dotted line in Fig. 4).

In addition, a significant main effect of the contextual average (the distance between context-setting items and the distant face in the test trial) was found, $F(1,32)=53.226, p<$ $.001, \eta^{2}=.625$, with a larger dissimilarity in far history sets (the continuous line is always above the dotted line in Fig. 4). We did not formulate any predictions about this main effect. Under the assumption that the context setting in these far history trials moves the prototype away from both the global average and the distant face on the test trial, this main effect suggests that the perceived difference between stimuli is increased for stimuli further from the norm.

Finally, in contrast to our predictions, no significant effect of the contextual trend was found, $F(1,32)=0.235, p=.631$, $\eta^{2}=.007$, nor did this factor change the asymmetry, $F(1,32)=$ $0.045, p=.834, \eta^{2}=.001$, nor did it interact with the contextual average in how that factor changed the asymmetry, $F(1,32)=0.687, p=.46, \eta^{2}=.021$. This indicates that the five most recent trials all have an equal influence on the development of the norm; that is, it is not the case that more recent items have a stronger effect on the norm.

\section{Discussion}

We reported findings on three topics of interest: the presence of asymmetry in similarity judgments and its relation to norm-based encoding, the role of the preceding trials on the position of this norm, and the role of recency within these preceding trials.

We found that when two faces are compared, the presentation order matters: If the face closest to the norm is presented second, similarity is judged as higher than when it is presented first. Importantly, in our experiment, one specific face could be a prototype for one participant, and a distant face for another; moreover, the prototype could be either closer to or further from the original face than the distant face. Taken together, the findings show that the asymmetry effect exists independently of the specific characteristics of the prototype. While previous experiments (e.g., Op de Beeck et al., 2003; Panis et al., 2011) already demonstrated this for abstract figures, we show that the same holds for faces. Consistent with the conclusions from this existing research, we conclude that the asymmetries reflect a norm that is built up during the course of the experiment through experience with the stimulus distribution.

Table 3 Dissimilarity ratings summary

\begin{tabular}{llll}
\hline Contextual Average & Contextual Trend & Presentation Order & Mean Dissimilarity Rating (SD) \\
\hline Close history & Trials move toward mid-face & Mid-face presented first & $3.44(S D 0.74)$ \\
& & Mid-face presented second & $3.36(S D 0.65)$ \\
& Trials move away from mid-face & Mid-face presented first & $3.36(S D 0.81)$ \\
Far history & & Mid-face presented second & $3.44(S D 0.79)$ \\
& Trials move toward mid-face & Mid-face presented first & $4.30(S D 0.90)$ \\
& & Mid-face presented second & $3.84(S D 0.90)$ \\
& & Mid-face presented first & $4.27(S D 0.98)$ \\
\end{tabular}


Table 4 Repeated measures general linear model results
$* p<.05$

$* * p<.01$

\begin{tabular}{lllll}
\hline Effect & $F$ & $d f$ & $\eta^{2}$ & $p$ \\
\hline Presentation order & 10.623 & 1 & .249 & $.003^{*}$ \\
Contextual average & 53.226 & 1 & .625 & $.000^{* *}$ \\
Contextual trend & 0.235 & 1 & .007 & .631 \\
Presentation order $\times$ contextual average & 11.633 & 1 & .267 & $.002^{*}$ \\
Presentation order $\times$ contextual trend & 0.045 & 1 & .001 & .834 \\
Contextual average $\times$ contextual trend & 0.560 & 1 & .017 & .460 \\
Pres. order $\times$ contextual average $\times$ contextual trend & 0.687 & 1 & .021 & .413 \\
\hline
\end{tabular}

We also observed a strong effect of the content of the context-setting trials. When the contextual average was exactly in the middle between the mid-face and the distant face on a test trial, the effect of asymmetry was abolished. Thus, five trials (10 stimuli) are enough to shift the norm toward the contextual average. There has been extensive previous research indicating context-dependent norm shifts (e.g., Carbon et al., 2007; Leopold et al., 2001; Leopold et al., 2005; Nishimura et al., 2008; Panis et al., 2011; Rhodes \& Jeffery, 2006; Rhodes, Maloney, Turner, \& Ewing, 2007); however, these studies usually did not take global exposure to the stimuli into account, nor did they control for the physical characteristics of the norm-as we did by counterbalancing stimuli across participants. One of the merits of our experimental setup is that the global exposure was kept constant by making sure participants had equal exposure to all presented stimuli. We find that independently of any total, long-term exposure, the norm is affected strongly by short-term exposure.

Additionally, we found that exposure to a certain set of stimuli increases subjective similarity between those stimuli, in line with Rhodes et al. (2007). It appears that perceived differences are smaller between stimuli close to the norm than between stimuli further from the norm, even if the actual difference between the stimuli is identical in both situations.

Furthermore, we found that in our paradigm, the temporal trend of the averages of recent trials (toward the mid-face or away from the mid-face) has no significant effect. This grants some insight into the time frame in which the context influences the norm. Our study used sets of five context-setting trials with 2 items each, making a total of 10 items. While we do find a strong effect of the last 10 items overall (see above), it appears that the order of these 10 items is irrelevant; that is, the most recent stimulus pair has the same influence on the norm as the first. Thus, effects of temporal context on the face norm are integrated across multiple trials/faces. This is in line with Panis et al. (2011), who found that learning takes place on a time scale longer than single successive trials. Note that these findings show a contrast with visual adaptation, which may occur after only a single trial. An interesting avenue for future research would be an experimental setup with varying lengths of context-setting trials and of intertrial intervals. This would increase insight into the time frame of contextdependent exposure of the norm.

Finally, although we interpret our results in the context of norm-based encoding, we reiterate the suggestion of Panis et al. (2011) that norm-based and exemplar-based encoding need not necessarily conflict. It seems possible that our brain uses a combination of the two to encode faces. For example, faces could be stored partly as exemplars, but on top of that, some information could be encoded in terms of their relation to a norm.

In sum, we showed how the norm used as a basis to encode faces is influenced by the faces presented on previous trials. The mid-face face is typically used as the norm, but a systematic manipulation of the average face in a relatively small set of preceding trials can shift this norm. Nevertheless, the position of this norm is not only influenced by the most

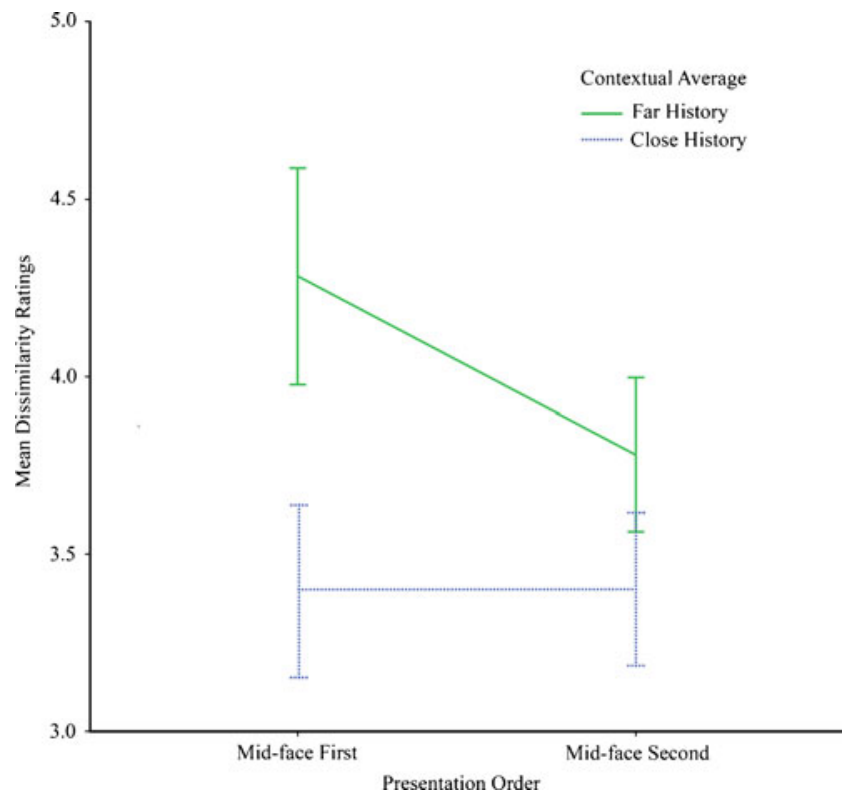

Fig. 4 Interaction between presentation order and contextual average. The interaction between the presentation order in the trial of interested (mid-face first or mid-face second) and the contextual average, that is, the distance between the context-setting items and the distant face in the trial of interest (close history versus far history). The error bars represent the standard error of the mean across participants 
recently presented face and is based upon an integration of multiple faces.

Author Note This research was supported by grant CDA-0040/2008 (Human Frontier Science Program) and G.0562.10 (Fund for Scientific Research FWO-Flanders).

\section{References}

Brainard, D. H. (1997). The psychophysics toolbox. Spatial Vision, 10, 433-436.

Carbon, C.-C., Strobach, T., Langton, S. R., Harsányi, G., Leder, H., \& Kovács, G. (2007). Adaptation effects of highly familiar faces: Immediate and long lasting. Memory \& Cognition, 35(8), 19661976.

Lee, K., Byatt, G., \& Rhodes, G. (2000). Caricature effects, distinctiveness, and identification: Testing the face-space framework. Psychological Science, 11(5), 379-385.

Leopold, D. A., Bondar, I. V., \& Giese, M. A. (2006). Norm-based face encoding by single neurons in the monkey inferotemporal cortex. Nature, 442(3), 572-575.

Leopold, D. A., O'Toole, A. J., Vetter, T., \& Blanz, V. (2001). Prototype-referenced shape encoding revealed by high-level aftereffects. Nature Neuroscience, 4(1), 89-94.
Leopold, D. A., Rhodes, G., Müller, K.-M., \& Jeffery, L. (2005). The dynamics of visual adaptation to faces. Proceedings of the Royal Society B, 272, 897-904.

Loffler, G., Yourganov, G., Wilkinson, F., \& Wilson, H. R. (2005). fMRI evidence for the neural representation of faces. Nature Neuroscience, 8(10), 1386-1390.

Nishimura, M., Maurer, D., Jeffery, L., Pellicano, E., \& Rhodes, G. (2008). Fitting the child's mind to the world: Adaptive norm-based coding of facial identity in 8-year-olds. Developmental Science, 11(4), 620-627.

Nosofsky, R. M. (1991). Stimulus bias, asymmetric similarity, and classification. Cognitive Psychology, 23(1), 94-140.

Op de Beeck, H., Wagemans, J., \& Vogels, R. (2003). Asymmetries in stimulus comparisons by monkey and man. Current Biology, 13, 1803-1808.

Panis, S., Wagemans, J., \& Op de Beeck, H. P. (2011). Dynamic normbased encoding for unfamiliar shapes in human visual cortex. Journal of Cognitive Neuroscience, 23(7), 1829-1843.

Rhodes, G., \& Jeffery, L. (2006). Adaptive norm-based coding of facial identity. Vision Research, 46, 2977-2987.

Rhodes, G., Jeffery, L., Watson, T. L., Clifford, C. W., \& Nakayama, K. (2003). Fitting the mind to the world: Face adaptation and attractiveness aftereffects. Psychological Science, 14(6), 558-566.

Rhodes, G., Maloney, L. T., Turner, J., \& Ewing, L. (2007). Adaptive face coding and discrimination around the average face. Vision Research, 47, 974-989.

Suzuki, S., \& Cavanagh, P. (1998). A shape-contrast effect for briefly presented stimuli. Journal of Experimental Psychology, 24(5), $1315-1341$

Tversky, A. (1977). Features of similarity. Psychological Review, 84(4), 327. 